To appear in the Astrophysical Journal, December 2007

\title{
The ACS Fornax Cluster Survey. II. The Central Brightness Profiles of Early-Type Galaxies: A Characteristic Radius on Nuclear Scales and the Transition from Central Luminosity Deficit to Excess ${ }^{1}$
}

\author{
Patrick Côté ${ }^{2}$, Laura Ferrarese ${ }^{2}$, Andrés Jordán ${ }^{3}$, John P. Blakeslee ${ }^{4}$, Chin-Wei Chen ${ }^{2,5}$, Leopoldo \\ Infante $^{6}$, David Merritt ${ }^{7}$, Simona Mei ${ }^{8}$, Eric W. Peng ${ }^{2}$, John L. Tonry ${ }^{9}$, Andrew A. West ${ }^{10}$, \\ Michael J. West ${ }^{11}$
}

\begin{abstract}
We analyse brightness profiles for 143 early-type galaxies in the Virgo and Fornax Clusters, observed with the Advanced Camera for Surveys on the Hubble Space Telescope. Sérsic models are found to provide accurate representations of the global profiles with a notable exception: the observed profiles deviate systematically inside a characteristic "break" radius of $R_{b} \approx 0.02_{-0.01}^{+0.025} R_{e}$, where $R_{e}$ is the effective radius of the galaxy. The sense of the deviation is such that bright galaxies $\left(M_{B} \lesssim-20\right)$ typically show central light deficits with respect to the inward extrapolation of the Sérsic model, while the great majority of low- and intermediate-luminosity galaxies $\left(-19.5 \lesssim M_{B} \lesssim-15\right)$ show central light excesses; galaxies of intermediate luminosities $\left(-20 \lesssim M_{B} \lesssim-19.5\right)$ are generally well fitted by Sérsic models over all radii. We show that the slope, $\gamma^{\prime}$, of the central surface brightness profiles, when measured at fixed fractions of $R_{e}$, varies
\end{abstract}

\footnotetext{
${ }^{1}$ Based on observations with the NASA/ESA Hubble Space Telescope obtained at the Space Telescope Science Institute, which is operated by the Association of Universities for Research in Astronomy, Inc., under NASA contract NAS 5-26555.

${ }^{2}$ Herzberg Institute of Astrophysics, National Research Council of Canada, Victoria, BC, V9E 2E7, Canada

${ }^{3}$ European Southern Observatory, Karl-Schwarzschild-Str. 2, 85748 Garching, Germany

${ }^{4}$ Department of Physics \& Astronomy, Washington State University, Pullman, WA 99164-2814

${ }^{5}$ Institute of Astronomy, National Central University Taiwan, 32054, Chungli, Taiwan

${ }^{6}$ Departamento de Astronomía y Astrofísica, Pontificia Universidad Católica de Chile, Santiago 22, Chile

${ }^{7}$ Department of Physics, Rochester Institute of Technology, 84 Lomb Memorial Drive, Rochester, NY 14623

${ }^{8}$ GEPI, Observatoire de Paris, Meudon Cedex, France

${ }^{9}$ Institute for Astronomy, University of Hawaii, 2680 Woodlawn Drive, Honolulu, HI 96822

${ }^{10}$ Department of Astronomy, University of California, 601 Campbell Hall, Berkeley, CA 94720

${ }^{11}$ Department of Physics \& Astronomy, University of Hawaii, Hilo, HI 96720; and Gemini Observatory, Casilla 603, La Serena, Chile
} 
smoothly as a function of galaxy luminosity in a manner that depends sensitively on the choice of measurement radius. We find no evidence for a core/power-law dichotomy, and show that a recent claim of strong bimodality in $\gamma^{\prime}$ is likely an artifact of the biased galaxy selection function used in that study. To provide a more robust characterization of the inner regions of galaxies, we introduce a parameter, $\Delta_{0.02}=\log \left(\mathcal{L}_{g} / \mathcal{L}_{s}\right)$ - where $\mathcal{L}_{g}$ and $\mathcal{L}_{s}$ are the integrated luminosities inside $0.02 R_{e}$ of the observed profile and of the inward extrapolation of the outer Sérsic model - to describe the central luminosity deficit $\left(\Delta_{0.02}<0\right)$ or excess $\left(\Delta_{0.02}>0\right)$. We find that $\Delta_{0.02}$ varies smoothly over the range of $\approx 720$ in luminosity spanned by the sample galaxies, with again no evidence for a dichotomy. We argue that the central light excesses in $M_{B} \gtrsim-19$ galaxies may be the analogs of the dense central cores predicted by some numerical simulations to form via gas inflows.

Subject headings: galaxies: clusters: individual (Virgo, Fornax)-galaxies: elliptical and lenticular, $\mathrm{cD}$-galaxies: nuclei: galaxies: structure

\section{Introduction}

Pioneering HST imaging studies of the centers of early-type galaxies suggested an apparently abrupt transition in central stellar density at $M_{B} \sim-20.3 \mathrm{mag}$ - the so-called "core/power-law dichotomy" (e.g., Ferrarese et al. 1994; Lauer et al. 1995). These findings prompted the widely held view that the bright ("core") and faint ("power-law") galaxies follow distinct evolutionary routes (e.g., Faber et al. 1997). However, the evidence for such a dichotomy has lessened (although not entirely disappeared) following more recent studies that identified a population of galaxies with intermediate properties (Rest et al. 2001; Ravindranath et al. 2001). The slope, $\gamma^{\prime}$, of the central surface brightness profile - usually parameterized as a "Nuker" law (essentially two power-laws that merge at a characteristic "break" radius; Lauer et al. 1995) and measured at the angular distance corresponding to the instrumental resolution - has traditionally been taken as a diagnostic of this behavior.

However, using ACS profiles for 100 early-type galaxies belonging to the Virgo Cluster, Ferrarese et al. (2006a) showed that Sérsic models provide more accurate parameterizations of the global brightness profiles than do Nuker models (see also Graham 2004; Ferrarese et al. 2006c) and argued that the core/power-law dichotomy is an artifact introduced in part by the use of an inappropriate (i.e., power-law) parameterization of the outer profiles, combined with a tendency in previous work (which usually relied on HST brightness profiles of limited radial extent) to not properly account for the compact stellar nuclei found in low- and intermediate luminosity galaxies (e.g., Graham \& Guzmán 2003; Grant et al. 2005; Côté et al. 2006).

In this paper, we use the best available imaging dataset - in terms of depth, radial coverage, angular resolution, completeness and homogeneity - to re-examine the central structure of early- 
type galaxies. Our analysis relies on HST/ACS imaging for 100 early-type members of the Virgo (previously discussed in Ferrarese et al. 2006ab and Côté et al. 2006) and new HST/ACS imaging for 43 early-type members of the Fornax cluster (Jordán et al. 2007). Our principle finding is clear evidence for a continuous, systematic progression from central luminosity deficit $\left(M_{B} \lesssim-20\right)$ to excess $\left(M_{B} \gtrsim-19\right)$ within a characteristic radius, approximately equal to $2 \%$ of the galaxy effective radius. We find no evidence for a "core/power-law" dichotomy.

\section{Observations}

HST images for 143 members of the Virgo and Fornax Clusters were acquired with the Advanced Camera for Surveys (ACS, Ford et al. 1998) as part of the ACS Virgo (ACSVCS; GO-9401) and Fornax (ACSFCS; GO-10217) Cluster Surveys (Côté et al. 2004; Jordán et al. 2007). Surface brightness fluctuation distance measurements (Mei et al. 2005, 2007; Blakeslee et al. 2008, in preparation) reveal the program galaxies to span luminosity ranges of $\approx 545$ (Virgo), $\approx 345$ (Fornax) and $\approx 720$ (combined). All galaxies have early-type morphologies (i.e., E, S0, dE, dE, N or dS0) and are confirmed velocity members of their respective clusters. Images were taken in the Wide Field Channel (WFC) mode with a filter combination (F475W and F850LP) roughly equivalent to the $g$ and $z$ bands in the Sloan Digital Sky Survey (SDSS) photometric system. The images cover a roughly $200^{\prime \prime} \times 200^{\prime \prime}$ field with $0^{\prime \prime}\left(05\right.$ pixel $^{-1}$ sampling and $\approx 0^{\prime \prime} 1$ resolution. This resolution limit translates to physical scales of 8.0 and 9.5 pc for Virgo and Fornax, respectively. For the 21 Virgo galaxies brighter than $B_{T}=12\left(M_{B} \approx-19.2\right)$, the profiles were extended in radius by matching the ACS profiles to those measured from SDSS $g$ and $z$ mosaics (Data Release 5; Adelman-McCarthy et al. 2007) generated using the procedures described in West et al. (2007). The ACSVCS sample is complete for early-type galaxies brighter than $B \approx 12\left(M_{B} \approx-19.2\right)$ and $44 \%$ complete down to its limiting magnitude of $B \approx 16\left(M_{B} \approx-15.2\right)$. The ACSFCS sample, meanwhile, is complete down to its limiting magnitude of $B \approx 15.5\left(M_{B} \approx-16.1\right)$ with the exception of a single galaxy $($ FCC161 $=$ NGC1379) for which the data acquisition failed because of a shutter problem.

Full details on the construction of the azimuthally averaged brightness profiles and the adopted fitting procedures (e.g., correction for dust obscuration, masking of background sources, the identification of offset nuclei via centroid shifts, and the choice of weighting schemes and minimization

routines, etc) are given in $\S \S 3.1,3.2$ and 3.3 of Ferrarese et al. (2006a) and $\S \S 3.1,3.2,4.1$ and 4.2 of Côté et al. (2006).

\subsection{Parameterization of the Brightness Profiles}

Figures 1 and 2 shows $g$ and $z$ surface brightness profiles for nine representative galaxies belonging to each of the ACSVCS and ACSFCS samples. To parameterize the brightness profiles, 
we begin by noting that Sérsic (1968) models

$$
I_{S}(R)=I_{e} \exp \left\{-b_{n}\left[\left(\frac{R}{R_{e}}\right)^{1 / n}-1\right]\right\},
$$

generally provide accurate descriptions of the global profiles of the program galaxies - most importantly the downward curvature on large scales - with only three free parameters $\left(I_{e}, n\right.$ and $R_{e}$ ). However, the central regions of the profiles (typically $R \lesssim 100 \mathrm{pc}$ for the brightest galaxies, and $R \lesssim 10 \mathrm{pc}$ for the faintest), deviate significantly from the inward extrapolations of the Sérsic profiles for both galaxies brighter than $M_{B} \lesssim-20$ (which show central light deficits) and for most galaxies fainter than $M_{B} \gtrsim-19.5$ (which usually show central excesses). By contrast, most galaxies with $-20 \lesssim M_{B} \lesssim-19.5$ are reasonably well fitted by a single Sérsic model over all radii, as previously noted by Graham \& Guzmán (2003). The discovery of a systematic progression from central light deficit to excess along the luminosity function was discussed extensively in Côté et al. (2006) and Ferrarese et al. (2006ab). This transition from deficit to excess at $-20.0 \lesssim M_{B} \lesssim-19.5$ corresponds to Sérsic indices of $3.9 \lesssim n \lesssim 3.5$, based on the scaling relations presented in $\S 4.3$ of Ferrarese et al. (2006a; see their Eq. 27).

To put this trend on a more quantitative footing, we parameterize the observed brightness profiles of the ACSVCS and ACSFCS galaxies in two different ways. First, given that the departures from Sérsic models always occur on scales of a few arcseconds and usually much less - meaning that the innermost behavior is dominated by the instrumental point spread function (PSF) a simple, PSF-convolved, power-law profile (with $0 \lesssim \gamma^{\prime} \lesssim 2$ ) inside a break radius, $R_{b}$, usually provides an adequate representation of the observed profiles. The short dashed curves in each panel of Figures 1 and 2 shows such "core-Sérsic" models (Graham et al. 2003),

$$
I_{c S}(R)=I^{\prime}\left[1+\left(\frac{R_{b}}{R}\right)^{\alpha}\right]^{\gamma / \alpha} \exp \left[-b_{n}\left(\frac{R^{\alpha}+R_{b}^{\alpha}}{R_{e}^{\alpha}}\right)^{1 /(\alpha n)}\right],
$$

fitted to the profiles in both bandpasses after convolution with the instrumental PSFs. Note that $I^{\prime}$ in Eq. (2) is related to the intensity, $I_{b}$, at the break radius $R_{b}$ through the relation:

$$
I^{\prime}=I_{b} 2^{-\gamma / \alpha} \exp \left[b_{n}\left(2^{1 / \alpha} R_{b} / R_{e}\right)^{1 / n}\right] .
$$

Thus, this choice of parameterization requires a total of five free parameters $\left(I_{b}, \alpha, \gamma, n\right.$ and $\left.R_{e}\right)$. For comparison, the dotted curves in each panel of Figures 1 and 2 show the inward extrapolations of an outer Sérsic component, illustrating the systematic evolution from deficit to excess. The arrows show the fitted values of $R_{b}$ for each galaxy.

As pointed out above, the profiles in the innermost regions are dominated by the instrumental PSF, so a different parameterization of the inner component could provide equally acceptable fits, particuarly for the low- and intermediate-luminosity galaxies. Indeed, a detailed study of the 


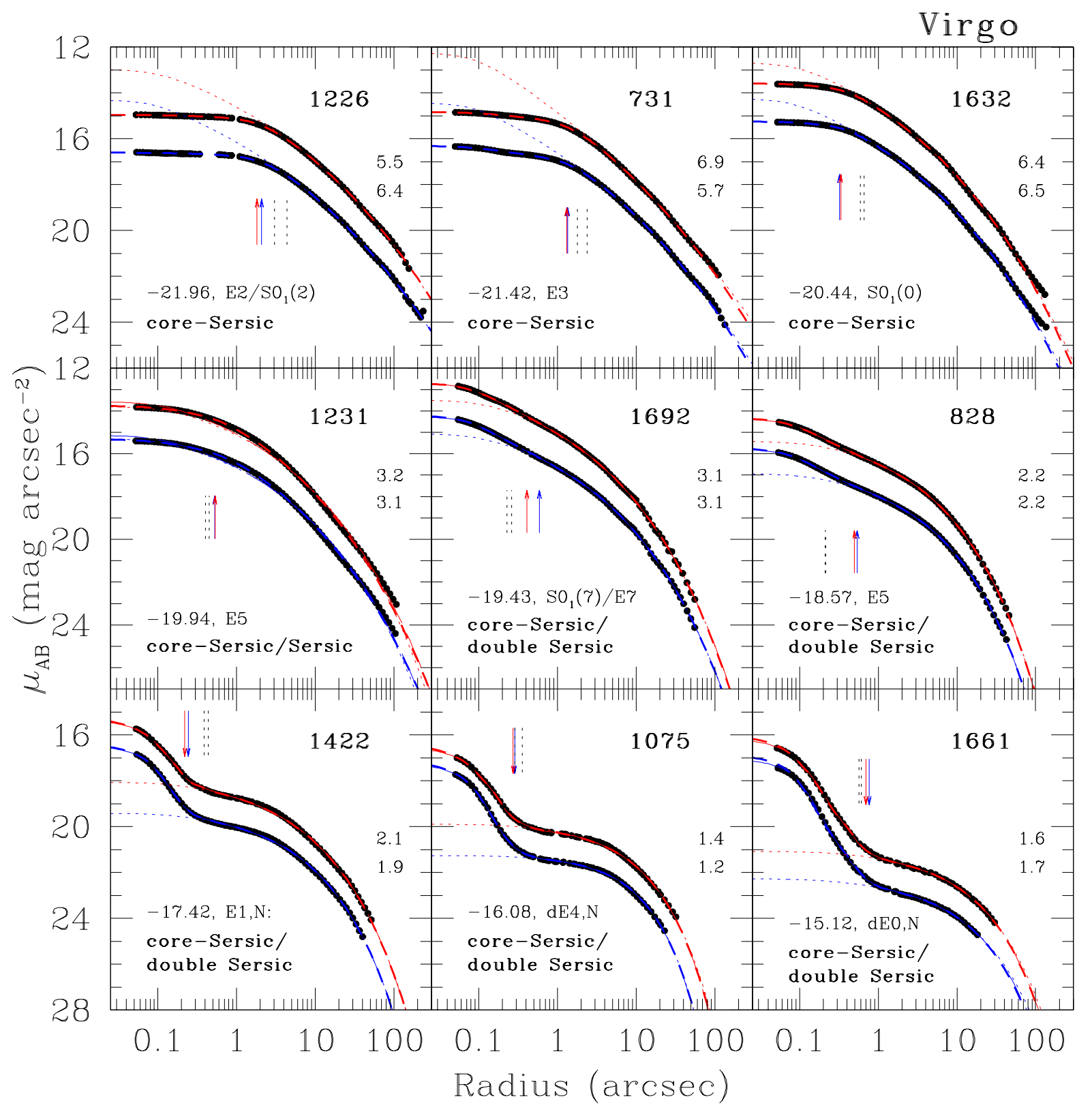

Fig. 1. - Surface brightness profiles for nine representative galaxies from the ACSVCS. Identification numbers from the Virgo Cluster Catalog (VCC) of Binggeli, Sandage \& Tammann (1985) are given in the upper right corner of each panel. For each of these galaxies, which span a range of $\approx 545$ in blue luminosity, we show both the $g$ and $z$ profiles as the lower and upper points, respectively. The galaxies are ordered according to decreasing absolute blue magnitude which is recorded in each panel, along with their morphological types and the best-fit Sérsic indices, $n$, for the galaxy measured in the $g$ and $z$ profiles (lower and upper labels). For each profile, we show the best-fit "core-Sérsic" (short dashed curves) and "composite" model (long dashed curves; see \$2.1). The red and blue arrows show the break radii, $R_{b}$, for the fitted core-Sérsic models (in $g$ and $z$, respectively) while the dotted vertical lines in each panel are drawn at $2 \%$ of the effective radius. The dotted curves show the inward extrapolations of the Sérsic component that best fits the profile for $R \gtrsim R_{b}$. Note the smooth transition from luminosity "deficit" to "excess" as one moves down 


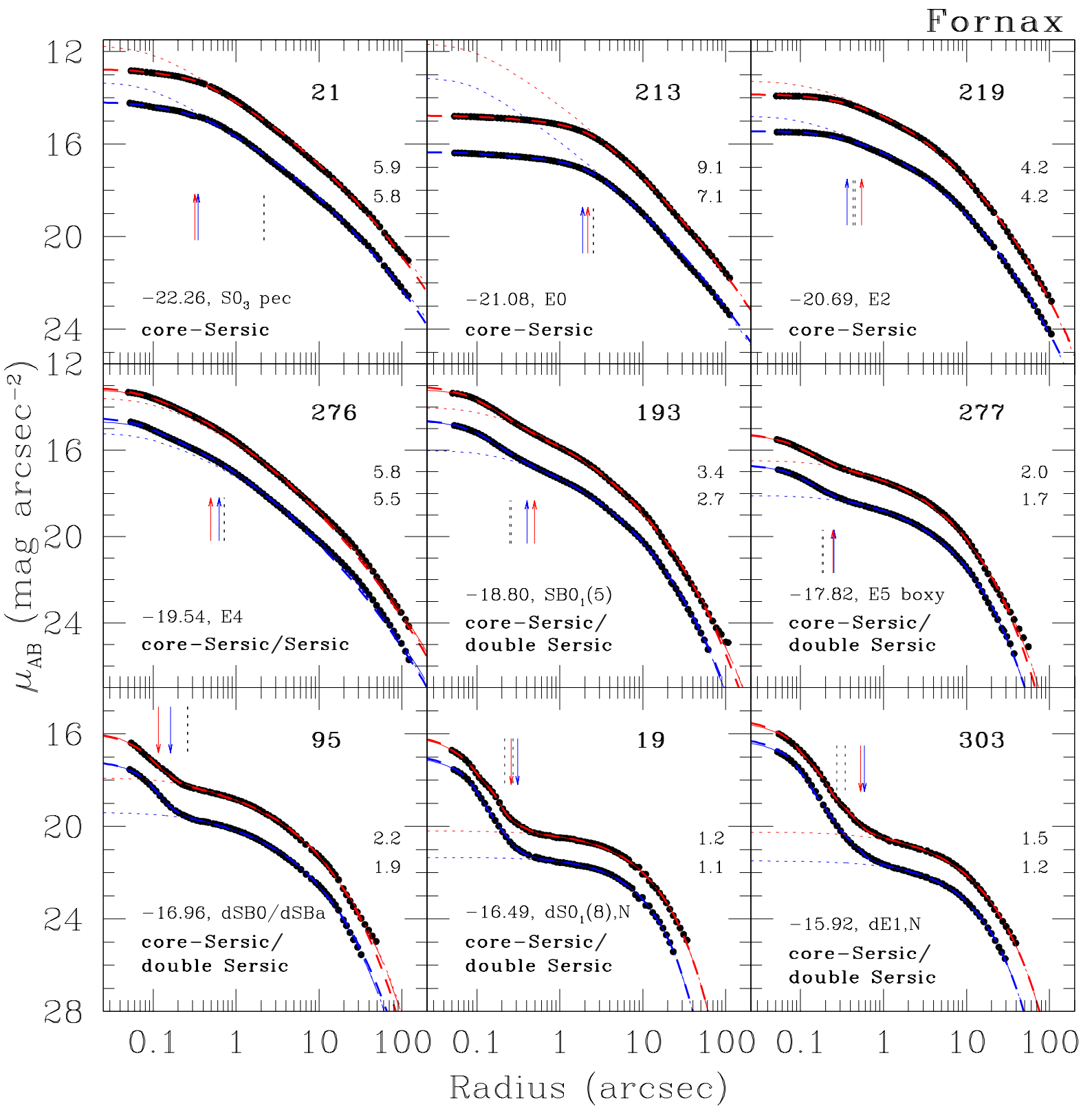

Fig. 2.- Same as in the previous figure, except for nine representative galaxies from the ACSFCS. Identification numbers from the Fornax Cluster Catalog (FCC) of Ferguson (1989). The galaxies, which span a range of $\approx 345$ in blue luminosity, exhibit the same basic trends as those in the Virgo Cluster. Note the small break radius for FCC21 (= NGC1316 = Fornax A), a peculiar S0 LINER/radio galaxy that shows clear evidence of recent merging (i.e., dust, $\mathrm{H} \alpha$ filaments, shells and ripples; Schweizer 1980). 
nuclear brightness profiles of nearby galaxies (Ferrarese et al. 2008, in preparation) suggests that an excellent parameterization over all scales is given by a double Sérsic model,

$$
\begin{aligned}
& I_{d S}(R)=I_{S_{1}}(R)+I_{S_{1}}(R) \\
& I_{d S}(R)=I_{e, 1} \exp \left\{-b_{n, 1}\left[\left(\frac{R}{R_{e, 1}}\right)^{1 / n_{1}}-1\right]\right\}+I_{e, 2} \exp \left\{-b_{n, 2}\left[\left(\frac{R}{R_{e, 2}}\right)^{1 / n_{2}}-1\right]\right\}
\end{aligned}
$$

in which one component corresponds to the galaxy profile and the other to the central light excess (i.e., a compact spheroidal or flattened stellar component). In this case, a total of six parameters are needed to describe the entire brightness profile $\left(I_{e, 1}, n_{1}, R_{e, 1}, I_{e, 2}, n_{2}\right.$ and $\left.R_{e, 2}\right)$.

Thus, for our second ("composite") description of the ensemble profiles we adopt, on an object-by-object basis, one of three parameterizations that best fits the observed profile: (1) a core-Sérsic model (Eq. 2); (2) a single, unbroken Sérsic model (Eq. 1); or (3) a double Sérsic model (Eq. 4). As mentioned above, the precise choice of parameterization is found to depend strongly on galaxy luminosity: all galaxies brighter than $M_{B} \lesssim-20$ are modeled with core-Sérsic laws, while most galaxies slightly fainter than this $\left(-20 \lesssim M_{B} \lesssim-19.5\right)$ are well fitted with a single Sérsic model over all radii. For the majority of galaxies fainter than $M_{B} \gtrsim-19.5$, a double Sérsic model can accurately match the profiles on all scales. A small number of galaxies $(\lesssim 10 \%$ of the sample) fainter than $M_{B} \approx-17.5$ are found not to require a second Sérsic component (i.e., they contain no obvious central nucleus and their profiles are generally well fitted by a single Sérsic model). We shall return to these interesting objects in \$4. The long dashed curves in Figures 1 and 2 show these "composite models", with the exact choice of parametrization listed in the lower left corner of each panel. The short and long dashed curves are generally indistinguishable, confirming that, for these particular galaxies, either approach yields an acceptable parameterization of the profiles on both large and small scales. Our conclusions are therefore robust to the choice of these two parameterizations.

\section{Results}

\subsection{Central Surface Brightness Profile Slopes}

While it is well established that the brightest early-type galaxies differ from their fainter counterparts in terms of isophotal shape, ellipticity, kinematics and stellar populations (see, e.g., Bender et al. 1989; Kormendy \& Djorgovski 1989; Caon et al. 1993; Ferrarese et al. 2006a; Emsellem et al. 2007) the strongest evidence for a bonafide dichotomy in their structural properties — rather

than a continous variation along the luminosity function with some intrinsic scatter - has come from the behavior of their central brightness profile slopes (Ferrarese et al. 1994; Lauer et al. 1995; Rest et al. 2001; Ravindranath et al. 2001; Lauer et al. 2007). However, Ferrarese et al. (2006ac) noted that such slope measurements have often relied on heterogeneous archival HST images (i.e., different instruments, filters, and resolution limits) or on galaxy samples with ill-defined selection 
functions and spanning wide ranges in distance (e.g., from $3.5 \mathrm{Mpc}$ to $\sim 320 \mathrm{Mpc}$ in the case of the recent study by Lauer et al. 2007). If such a dichotomy has a physical origin, then it would seem more appropriate to measure the slope at either the same physical - rather than angular radius, as was done in Ferrarese et al. (2006a), or at a point corresponding to a constant fraction of some characteristic scale radius in every galaxy (see also Ferrarese et al. 2006c).

Panels $(a-d)$ of Figure 3 illustrate this point by plotting the slope of the best-fit composite model at differing fractions of the effective radius of the galaxy $(0.005,0.01,0.05$ and 0.30$)$ as a function of absolute blue magnitude 1 That is to say, for each galaxy in our sample, we measure the instantaneous slope of the fitted models using the relations

$$
\begin{aligned}
\gamma_{\mathrm{CS}}^{\prime} & =\frac{d \log I_{c S}}{d \log R}=\frac{\gamma}{\left(R / R_{b}\right)^{\alpha}+1}+\frac{b_{n}}{n}\left(\frac{R}{R_{e}}\right)^{\alpha}\left[\frac{R^{\alpha}+R_{b}^{\alpha}}{R_{e}^{\alpha}}\right]^{1 /(\alpha n)-1} \\
\gamma_{\mathrm{S}}^{\prime} & =\frac{d \log I_{S}}{d \log R}=-\frac{b_{n}}{n}\left(\frac{R}{R_{e}}\right)^{1 / n} \\
\gamma_{\mathrm{dS}}^{\prime} & =\frac{d \log I_{d S}}{d \log R}=\frac{1}{I_{S_{1}}(R)+I_{S_{2}}(R)}\left[I_{S_{1}}(R) \frac{b_{n, 1}}{n_{1}}\left(\frac{R}{R_{e, 1}}\right)^{1 / n_{1}}+I_{S_{2}}(R) \frac{b_{n, 2}}{n_{2}}\left(\frac{R}{R_{e, 2}}\right)^{1 / n_{2}}\right]
\end{aligned}
$$

where the cS, S and dS subscripts refer to the core-Sérsic, Sérsic and double-Sérsic parameterizations discussed in 92.1 . Note that this approach differs from the one adopted by Ferrarese et al. (2006a) who - recognizing that compact stellar mass concentrations become increasingly prominent among progressively fainter and lower surface brightness galaxies - preferred to measure the slope of the underlying galaxy component rather than using the combined (i.e., galaxy + nucleus) profiles. The photometric and structural scaling relations derived by Ferrarese et al. (2006a) corroborated previous reports of a continuum in galaxy properties fainter than $M_{B} \approx-20$ (e.g., see $\S 4$ and 5 of Ferrarese et al. 2006a and references therein), with no dichotomy between giants and dwarfs (see also the discussion in $\S 1$ of Graham \& Guzman 2003).

Panels $(a-d)$ show the $\gamma^{\prime}-M_{B}$ relations found when the profiles are parameterized as described in $\S 2$ : a core-Sérsic profile (Eq. 2) for the brightest galaxies, a double Sérsic profile (Eq. 4) for most of the low- and intermediate-luminosity galaxies, and a Sérsic profile in all other cases (mostly intermediate luminosity and a few low-luminosity galaxies). Four important points are illustrated in these panels. First, the overall trends defined by the Virgo and Fornax galaxies are indistinguishable. Second, the behavior of the $\gamma^{\prime}-M_{B}$ relation 2 is obviously sensitive to the exact choice

\footnotetext{
${ }^{1}$ Absolute magnitudes for the ACSVCS galaxies are computed from the apparent magnitudes given in Côté et al. (2004), reddenings as described in Jordán et al. (2004) and SBF distances from Mei et al. (2007). For the 11 Virgo galaxies lacking SBF distances, we assume $(m-M)=31.09$ mag. For the ACSFCS galaxies, we use the apparent magnitudes and reddenings from Jordán et al. (2007) and SBF distances from Blakeslee et al. (2008, in preparation). Four ACSFCS galaxies without SBF distances are assigned the median Fornax distance.

${ }^{2}$ We present these scaling relations in terms of $M_{B}$ for two reasons. First, the scaling relations and dichotomies discussed in this paper have traditionally been expressed in this bandpass. Second, placing the ACSVCS and ACSFCS galaxies in the broader context of their cluster environments (i.e., see 3.2 is most straighforward using this bandpass since the wide-field, photographic (blue emulsion) surveys of Binggeli, Sandage \& Tammann (1985) and Ferguson
} 


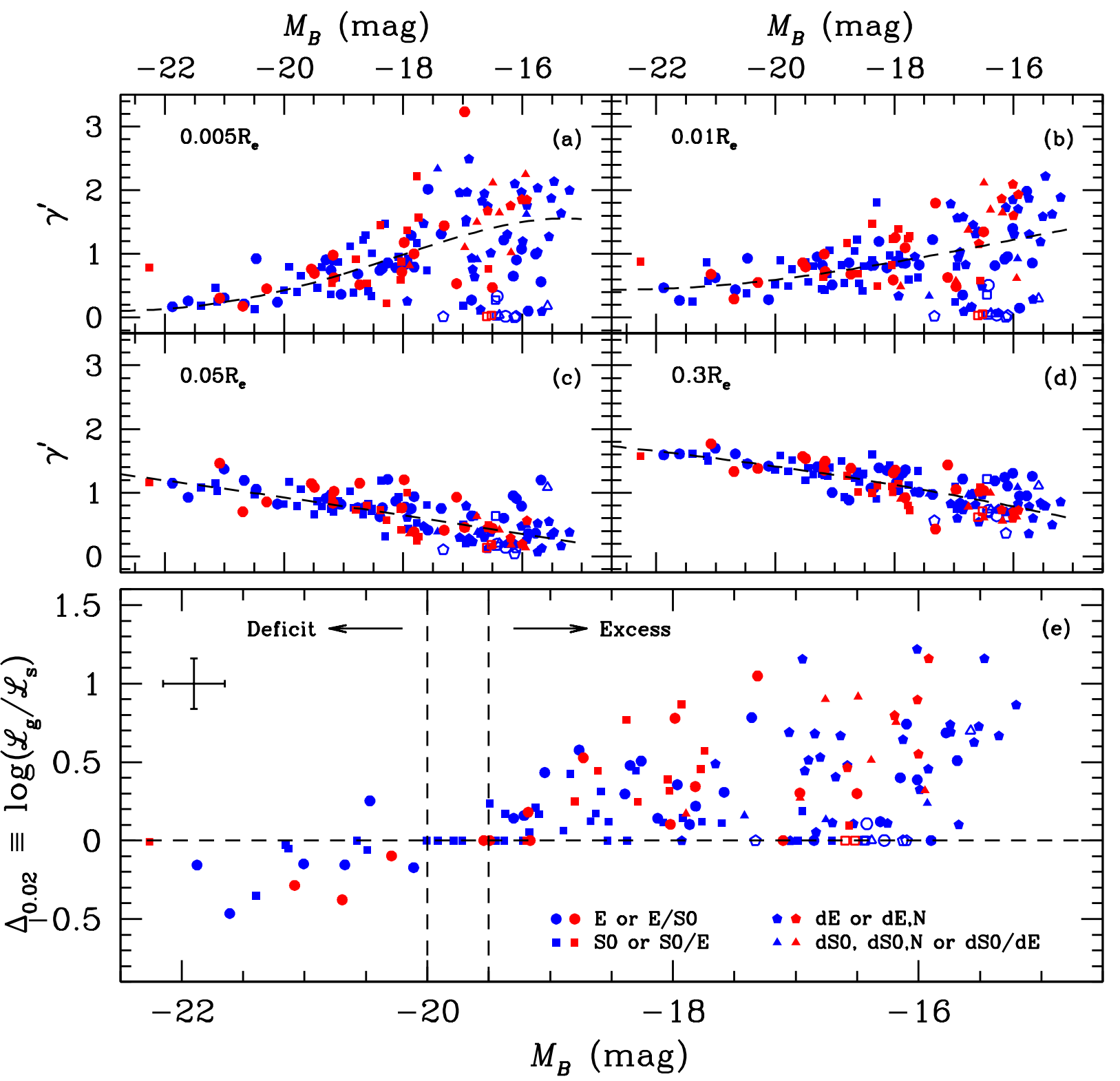

Fig. 3.- (Panels a-d) Slope of the best-fit composite model, $\gamma^{\prime}$, measured at different fractions of $R_{e}$ (i.e., 0.005, 0.01, 0.05 and 0.30). Virgo and Fornax galaxies are shown by blue and red symbols, respectively. The behavior of the $\gamma^{\prime}-M_{B}$ relation depends sensitively on the radius at which $\gamma^{\prime}$ is measured, but there is no evidence for a "core/power-law" dichotomy. The dashed curves show low-order Legendre polynomials that highlight the trends with magnitude. (Panel e) Dependence of $\Delta_{0.02}=\log \left(\mathcal{L}_{g} / \mathcal{L}_{s}\right)$ on galaxy magnitude for the composite fits (see text for details). Galaxies with central luminosity deficits have $\Delta_{0.02}<0$ while those with central excesses have $\Delta_{0.02}>0$. There is a smooth transition from central deficit to excess with decreasing galaxy luminosity. A typical errorbar is shown on the left side of the panel. Open symbols denote those galaxies with dE/dIrr transition morphologies, dust, young stellar clusters and/or evidence of young stellar populations from blue integrated colors. 
of measurement radius, particularly on the smallest angular scales. Third, the $\gamma^{\prime}-M_{B}$ relation undergoes an unmistakable "inversion" between $\sim 0.01-0.05 R_{e}$ : on the smallest scales, the profiles are found to steepen systematically as one moves down the luminosity function, whereas on larger scales, the profiles become progressively shallower with decreasing luminosity. And, finally, in no case is there clear evidence for a dichotomy or bimodality (see also Figures 1 and 2).

\subsection{Comparison to Lauer et al. (2007)}

The continuity of the $\gamma^{\prime}-M_{B}$ relations shown in the first four panels of Figure 3, while consistent with the finding of no core/power-law dichotomy by Ferrarese et al. (2006a), is apparently at odds with a recent detection of strong bimodality in $\gamma^{\prime}$ by Lauer et al. (2007). However, it is clear from this figure that the distribution of slopes found for any sample of galaxies will depend sensitively on both the choice of measurement radii and the luminosity distribution of the galaxies themselves.

In the upper panel of Figure 4, we plot as the open blue histogram the luminosity function, $\phi_{\text {gal }}$, of the 143 galaxies that make up the ACS Virgo and Fornax Cluster Surveys. The single- and double-hatched histograms show the results for the ACSVCS and ACSFCS samples, respectively. The dashed red curve shows a Schechter function with $\alpha=-1.40$ and $B^{*}=9.8\left(M_{B}^{*}=-21.4\right)$, the best-fit parameters for early-type $(\mathrm{E}+\mathrm{S} 0+\mathrm{dE}+\mathrm{dS} 0)$ galaxies in the Virgo Cluster according to Sandage, Binggeli \& Tammann (1985). The normalization of the Schechter function has been chosen to match the luminosity function of the ACSVCS and ACSFCS sample galaxies brighter than $M_{B} \approx-19$. Recall that both surveys are complete above this level and that the ACSFCS sample is complete for $M_{B} \lesssim-16.1$.

The blue histogram in panel (b) shows the luminosity distribution of the 219 galaxies analysed by Lauer et al. (2007) 3 The Schechter function from panel (a) is reproduced as the dashed red curve. The common element in the selection of these galaxies was the availability in the literature of Nuker model fits to brightness profiles derived from either WFPC1, WFPC2, NIC2 or NIC3 imaging. Two important properties of the Lauer et al. (2007) sample are worth noting. First, it is unrepresentative of the Schechter function form that provides a reasonable match to the luminosity distribution of the early-type galaxy populations in Virgo and Fornax in particular (Sandage et al. 1985; Ferguson \& Sandage 1988) and, more generally, those of galaxies in both cluster or field enviroments (e.g., Schechter 1976; Loveday et al. 1992; Marzke et al. 1994; Blanton et al. 2003). Second, as Lauer et al. (2007) point out, their sample is bimodal in luminosity - a result that we confirm. A

(1989) remain the most homogeneous photometric catalogs for Virgo and Fornax cluster galaxies. Homogeneous 'curve-of-growth' photometry from wide-field ugrizJHK imaging for the ACSVCS galaxies will be presented in Chen et al. (2008, in preparation).

${ }^{3}$ For comparison to the ACSVCS and ACSFCS samples, we have converted the $M_{V}$ magnitudes given in Lauer et al. (2007) to $M_{B}$ by assuming $(B-V)=0.96$ for BCG/E galaxies and $(B-V)=0.85$ for S0 galaxies (Fukugita, Shimasaku \& Ichikawa 1995). A color of $(B-V)=0.85$ was also adopted for the lone Sa galaxy in their sample. 
KMM analysis (McLachlan \& Basford 1988; Ashman, Bird \& Zepf 1994) indicates a 0.3\% chance that these data are drawn randomly from a unimodal Gaussian distribution (the null hypothesis). Assuming homoscedasticity and clipping seven galaxies with $M_{B}>-17.5$ (which serve to make the distribution even more bimodal), we find peaks at $M_{B}=-21.68$ and -19.30 .

What then is the expected distribution of $\gamma^{\prime}$ values for this dataset? As panels (a-d) of Figure 3 demonstrate, the detailed form of the $\gamma^{\prime}$ distribution will depend not only on the program galaxy magnitudes, but also on the choice of radius, $R / R_{e}$, at which $\gamma^{\prime}$ is measured. Lauer et al. (2007) choose to measure $\gamma$ at the data resolution limit $\left(0{ }^{\prime} \cdot 02,0{ }^{\prime} \cdot 04\right.$ or $0{ }^{\prime} \cdot 1$ depending on the instrument) for all galaxies in their sample, regardless of their distance or effective radius. According to the data in their Tables 1 and 2, R/Re thus varies between 0.00017 and 0.030 (a factor of 178) for the 150 galaxies in their sample for which estimates of $R_{e}$ are available in their Table 1 . To illustrate the behavior of the $\gamma^{\prime}$ distribution expected for their sample, we combine their $M_{B}$ values, calculated as described above, with the $\gamma^{\prime}-M_{B}$ relation shown as the smooth polynomial in panel (a) of Figure 3 , i.e., for $R / R_{e}=0.005$, which falls within the range of $R / R_{e}$ values used by Lauer et al. (2007). To approximate intrinsic scatter and measurement uncertainties, we assume a fixed dispersion of $\sigma\left(\gamma^{\prime}\right)$ $=0.1$ for $M_{B}<-22$, and rising linearly with galaxy magnitude to $\sigma\left(\gamma^{\prime}\right)=0.5$ at $M_{B}=-16$.

The $\gamma^{\prime}$ distribution found in a typical simulation is shown by the heavy magenta histogram in panel (c). In this case, KMM identifies highly significant peaks at $\gamma^{\prime} \approx 0.17$ and 0.70 , with a less than $0.1 \%$ chance of drawing these data from a unimodal Gaussian distribution. Thus, the simulated distribution is found to be strongly bimodal despite the fact that the input $\gamma^{\prime}-M_{B}$ varies smoothly along the luminosity function. The peak values of the simulated distribution should be compared to the values of 0.11 and 0.70 that we find when applying KMM to the actual $\gamma^{\prime}$ measurements tabulated in Table 2 of Lauer et al. (2007), reproduced here as the thin blue histogram. Although the two distributions differ in the details (which is not surprising since, as mentioned above, Lauer et al. do not measure $\gamma^{\prime}$ at a fixed fraction of $R_{e}$ or any other fixed physical scale), our exercise suggests that the bimodality in $\gamma^{\prime}$ reported by Lauer et al. (2007) is likely an artifact of a sample defined by a bimodal luminosity distribution that is: (1) unrepresentative of real galaxy ensembles; and (2) strongly biased in favor of bright, but intrinsically rare, galaxies that are known to have nearly constant-density cores. For instance, 56 of the galaxies in the Lauer et al. compilation (26\% of their sample) are brighter than VCC1226 (M49 = NGC4472), the brightest member of the Virgo Cluster.

\subsection{A Characteristic Radius: $R_{b}$}

To first order, Sérsic models provide accurate representations of the global, azimuthally averaged brightness profiles for almost all galaxies in our sample, largely independent of luminosity, morphological type, prior classification as giant or dwarf, and the presence or absence of morphological pecularities such as rings, shells and bars. However, the central deviations provide a notable exception to this rule. It is striking that, in all galaxies, the inner departure from the Sérsic model 

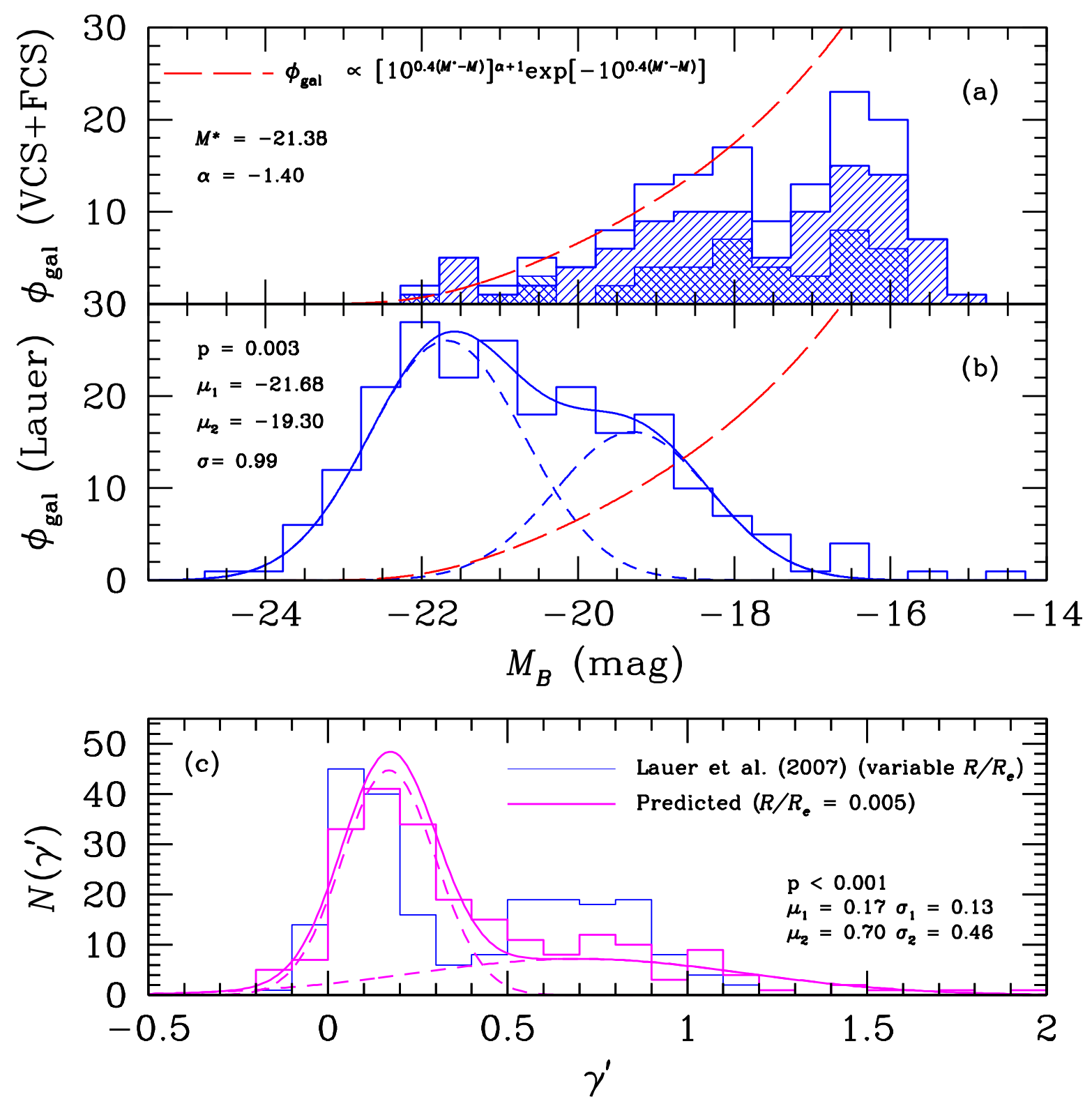

Fig. 4.- (Panel a) Luminosity function, $\phi_{\text {gal }}$, for the combined sample of 143 early-type galaxies from the ACS Virgo and Fornax Cluster Surveys (histogram). The dashed red curve shows a Schechter function with parameters $\alpha=-1.40$ and $B^{*}=9.8\left(M_{B}^{*} \approx-21.4\right)$, appropriate for $\mathrm{E}+\mathrm{S} 0+\mathrm{dE}+\mathrm{dS0}$ galaxies in the Virgo Cluster (Sandage, Binggeli \& Tammann 1985). The normalization is chosen to match the program galaxy luminosity function at $M_{B} \lesssim-19$ since both the Virgo and Fornax samples are complete above this luminosity. (Panel b) Luminosity function for the 219 galaxies in the compilation of Lauer et al. (2007). The sample is strongly non-representative of a Schechter function (the dashed curve from panel (a) is reproduced for comparison). A KMM analysis shows the sample to be bimodal in luminosity, with peaks at $M_{B}=-21.68$ and -19.30 , and a $0.3 \%$ chance of drawing these data randomly from a unimodal Gaussian distribution (the null hypothesis). The dotted and solid curves show the separate Gaussians, and their sum, as determined by KMM. (Panel c) A comparison of the distribution of $\gamma^{\prime}$ values measured by Lauer et al. (2007) (thin blue histogram) with that expected (magnenta histogram) based on their program galaxy luminosities and the $\gamma^{\prime}-M_{B}$ relation measured at $R=0.005 R_{e}$ (see Figure 3, panel a). KMM reveals there to be a less than $0.1 \%$ chance of drawing these data randomly from a unimodal Gaussian distribution. 
occurs at very nearly the same fraction of the effective radius. Using the core-Sérsic parameterization (which can be applied uniformly to all galaxies) we find the median logarithmic ratio of the break radius to the effective radius to be $\left\langle\log \left(R_{b} / R_{e}\right)\right\rangle=-1.68$ with $\mathrm{rms}$ scatter 0.33 . Thus, the inner deviations from the Sérsic law are found to occur at a characteristic radius of $R_{b} \sim 0.02 R_{e}$, with some scatter (the $\pm 1 \sigma$ range is $0.010-0.045$ ) that does not appear to correlate with any galaxy property. As an illustration, the vertical dashed lines in each panel of Figures 1 and 2 are drawn at $0.02 R_{e}$, where $R_{e}$ is measured independently in the $g$ and $z$ profiles.

\subsection{Light Deficits and Excesses: The $\Delta_{0.02}$ Parameter}

Because the departures from the inward extrapolation of the outer Sérsic component occur at $R_{b} \approx 0.02 R_{e}$, we define a parameter, $\Delta_{0.02}$, as the logarithm of the ratio of the total luminosity of the best-fit galaxy model (core-Sérsic or composite), $\mathcal{L}_{g}$, inside this radius to that of the outer Sérsic component in this same region: $\Delta_{0.02}=\log \left(\mathcal{L}_{g} / \mathcal{L}_{s}\right)$. Note that this description of the central behavior of the brightness profiles has the advantage relative to $\gamma^{\prime}$ that it is an integral measurement, rather than a differential one, and is thus less susceptible to noise in the profiles, PSF uncertainties, and the extent to which the stellar nucleus may be resolved. Galaxies with central light deficits then have $\Delta_{0.02}<0$, those with excesses have $\Delta_{0.02}>0$, and those whose profiles are well represented by Sérsic models over the full range in radius have $\Delta_{0.02}=0$.

Panel (e) of Figure 3 shows the dependence of $\Delta_{0.02}$ on absolute blue magnitude for the sample galaxies. The overall trends exhibited by the Virgo and Fornax galaxies are the same: a gradual transition from light deficits $\left(-0.5 \lesssim \Delta_{0.02} \lesssim 0\right)$ for galaxies brighter than $M_{B} \approx-20$ to light excesses $\left(0 \lesssim \Delta_{0.02} \lesssim 1.2\right)$ for the faintest program galaxies. This description of the inner galaxy also allows a straightforward definition of nucleated vs. non-nucleated galaxies. For instance, adopting $\Delta_{0.02}>0$ as the definition of a central stellar nucleus, one finds $69 \%$ of the combined sample galaxies, and $82 \%$ of all galaxies fainter than $M_{B} \approx-19$, to be nucleated. These estimates are consistent with the values for ACSVCS galaxies reported in Côté et al. (2006).

We point out that the tendency for early-type galaxies of low- and intermediate luminosity to have central brightness profiles that fall above Sérsic laws is not a new result: several previous investigators had noted that such behavior in isolated galaxies or using small datasets (e.g., Binggeli \& Jerjen 1998; Kormendy 1999; Stiavelli et al. 2001; Graham \& Guzmán 2003). However, the systematic variation along the luminosity function from central light deficit to light excess — with no apparent structural dichotomies between "core" and "power-law" galaxies at $M_{B} \approx-20$, nor between giants and dwarfs at $M_{B} \approx-18$ (c.f. Kormendy 1985) - has only been possible thanks to the uniformity, homogeneity and luminosity coverage of the ACSVCS and ACSFCS datasets. 

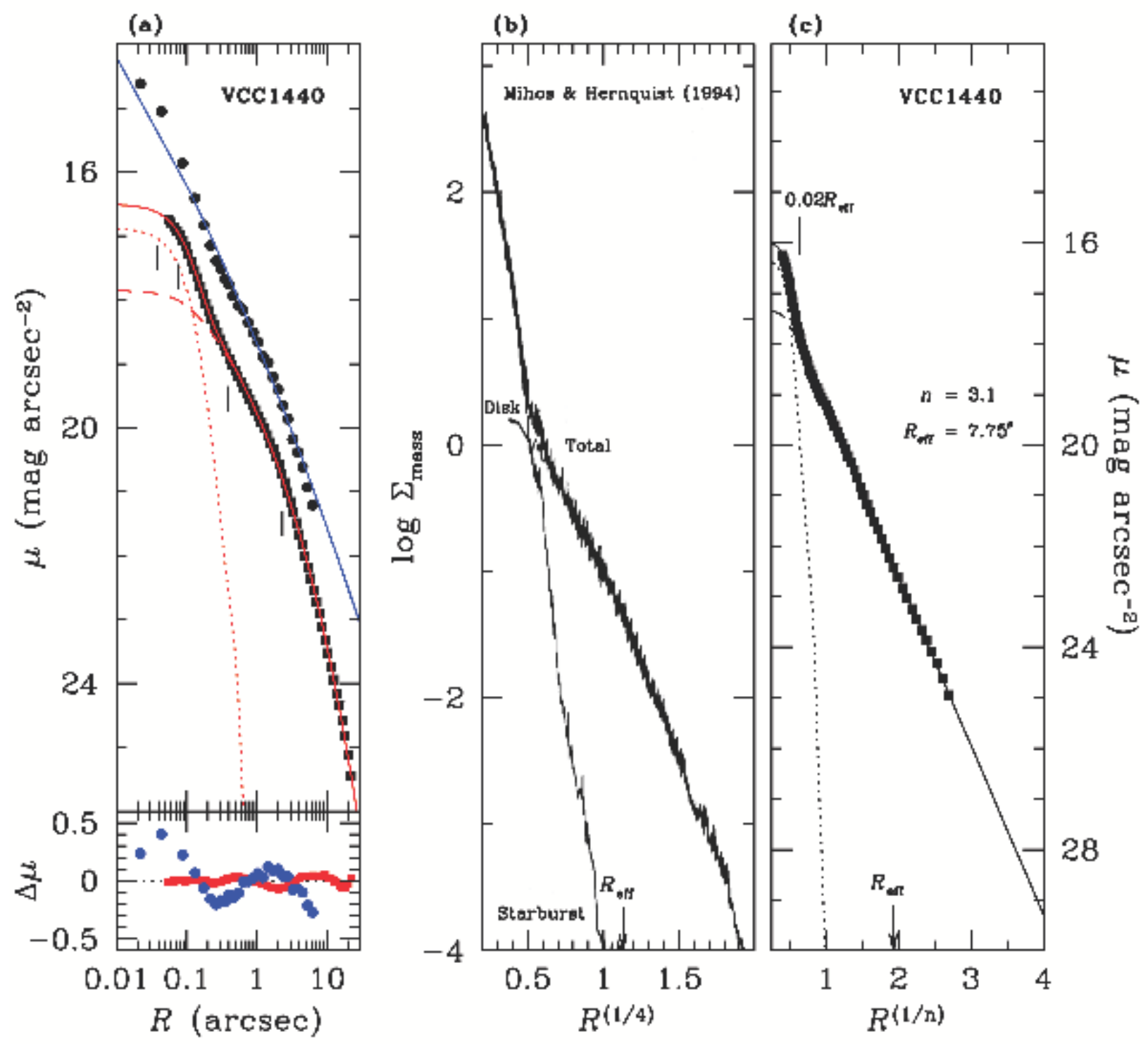

Fig. 5. - (Panel a) Comparison of WFPC1 and ACS/WFC brightness profiles for VCC1440, an E0 galaxy $\left(M_{B}=-15.94\right)$ previously classified as having a "power-law" profile (Lauer et al. 1995; 2007). The upper datapoints show the deconvolved, major axis, F555W WFPC1 profile of Lauer et al. (1995) and their fitted Nuker model. The lower datapoints show the azimuthally averaged ACS F475W profile along with the PSF-convolved, double Sérsic model (dashed and dotted curves) whose sum (solid red curve) best fits the observed profile. The datasets and models have been shifted apart by 0.75 mag for clarity. Residuals for both parameterizations are shown in the lower panel; note the S-shaped residuals for the Nuker model, indicative of a central nucleus (blue symbols; see also Ferrarese et al. 2006c). (Panel b) Predicted luminous mass profiles for the remnants of merging disk/halo galaxy models, showing the "dense stellar core" that forms as a result of gas dissipation and star formation. Panel (b) is adapted from the data/simulations shown in Figure 1a of Mihos \& Hernquist (1994). (Panel c) Alternate presentation of the ACS brightness profile for VCC1440, showing the excess light inside $\approx 0.02 R_{e}\left(\Delta_{0.02}=0.387 \pm 0.006\right)$. Compare with panel (b). In panels (b) and (c), the abscissa spans the same range when normalized to $R_{e}\left(10^{-3} \lesssim R / R_{e} \lesssim 10\right)$. 


\section{Discussion}

The central deficits in bright ellipticals have been traditionally interpreted as evidence for core evacuation by coalescing supermassive black hole (SBH) binaries (Ebisuzaki et al. 1991; Faber et al. 1997; Milosavljević \& Merritt 2001), although at least one recent study suggests that the dissipationless collapse of initially cold stellar distributions in existing dark matter haloes can also give rise to such features (Nipoti et al. 2006). The discovery of a systematic transition from central deficit to excess should provide an important new clue to the formation of both highand low-luminosity early-type galaxies and the connection to SBHs. It is worth noting that the mass fraction contributed by the central nuclei in low- and intermediate-luminosity galaxies is indistinguishable $(\sim 0.2 \%)$ from that of SBHs in bright galaxies, suggestive of a shared formation path (Côté et al. 2006, Ferrarese et al. 2006ab; Wehner \& Harris 2006; Rossa et al. 2006). On the other hand, McLaughlin \& King (2006) point out that feedback by stellar winds and supernovae may also be capable of reproducing the observed scaling relations without a direct connection between the nuclei and SBHs. And, as Ferrarese et al. (2006b) note, there are at least some galaxies (such as M32) that contain both a central excess (Worthey 2004) and a SBH (Verolme et al. 2002).

A lengthy discussion of the selection effects that prevented the ubiquitous nature of these nuclei from being fully appreciated with ground-based imaging was presented in Côté et al. (2006). Not surprisingly, the foremost advangtage of HST/ACS in this regard is its exceptional angular resolution. One might think, then, that the detection of nuclei in low- and intermediate-luminosity would have been straightforward in early high-resolution HST imaging, but this often proved not to be the case. For instance, the left panel of Figure 5 compares our ACS profile for VCC1440 a low-luminosity elliptical previously classified as a "power-law" galaxy - to the WFPC1 profile of Lauer et al. $(1995,2007) 4$ In this case, the limited radial extent of the WFPC1 profile, and the adopted parameterization of the galaxy profile as a power-law, allowed the full extent of the central excess to go unrecognized, although its signature is evident in the S-shaped residuals of the fitted Nuker model (see also Ferrarese et al. 2006c).

In hindsight, the realization that compact stellar mass concentrations reside at the centers of most low- and intermediate galaxies should perhaps have come as no surprise. Many theoretical studies have shown that such mass concentrations may be a generic outcome of the galaxy formation process. Proposed formation routes include repeated mergers of star clusters drawn to the galaxy center via dynamical friction, the growth of $\rho \propto r^{-7 / 4}$ density cusps due to two-body relaxation of stars orbiting a central SBH (Bahcall \& Wolf 1976) and/or central star formation driven by gas inflows - likely modulated by stellar evolution, mergers, IGM confinement, and feedback from stellar winds, supernovae or pre-existing AGN (e.g., Barnes \& Hernquist 1991; Mihos \& Hernquist 1994; McLaughlin et al. 2006; Li et al. 2007; Hopkins et al. 2007).

\footnotetext{
${ }^{4}$ Lauer et al. (2007) report $\gamma^{\prime}=0.89$ for this galaxy. We measure $\left\langle\gamma^{\prime}\right\rangle=1.08,1.40,0.79$ and 1.31 at $R / R_{e}=$ $0.005,0.01,0.05$ and 0.3 , respectively. These radii are indicated by the tickmarks in Figure 5 .
} 
A comparison of the properties (e.g., sizes, luminosity functions, mass fractions, colors) of nuclei and star clusters in ACSVCS program galaxies argues against the first scenario as the dominant formation mechanism (e.g., see $§ 5.2$ of Côté et al. 2006). Likewise, Bahcall-Wolf "cusps" generated by SBHs do not seem viable since most of the nuclei are resolved and thus much more extended than the predicted cusps (e.g., Merritt \& Szell 2006). On the other hand, gas inflow models either internally or externally modulated - seem more attractive as they have long predicted the formation of "dense stellar cores" in early-type galaxies. Consider the center and right panels of Figure 5 which compare the brightness profile of VCC1440 (the same galaxy shown in the left panel) to the simulations of Mihos \& Hernquist (1994). These authors showed that compact central starbursts are found in the remnants of disk/halo mergers and went on to argue that this central component would lead to "a break in the mass profile at small radii $\left(\sim 2 \% R_{e}\right)$ ". As Figure 5 and the discussion in 3.3 show, this may turn out to be a remarkably prescient prediction. The fact that the deviations from the fitted Sérsic models occur on roughly the same $\left(\sim 2 \% R_{e}\right)$ scale for both the central excesses and central deficits (which are usually attributed to binary SBH evolution; see above) presents something of a puzzle since it is unclear why these two unrelated physical mechanisms would give rise to deviations on the same (fractional) scale.

In any case, it will be important to test the gas inflow scenario envisaged by Mihos \& Hernquist (1994) by measuring star formation and chemical enrichment histories for the central regions of bright (deficit) and faint (excess) galaxies alike. Both the measured color profiles (Ferrarese et al. 2006a) and the model-dependent integrated colors (Côté et al. 2006) of the nuclei and galaxies in the ACSVCS suggests that stellar population differences between the two components may indeed exist, with the nuclei in the faintest systems generally being bluer than the underlying galaxy. On the other hand, at least some intermediate-luminosity galaxies show evidence for surprisingly red central components (e.g., $g-z \gtrsim 1.5$ ).

Finally, we point out that the open symbols in panel (e) of Figure 3 denote galaxies with "dE/dIrr transition" morphologies: i.e., dust, young stellar clusters and/or evidence of young stellar populations from blue integrated colors (Ferrarese et al. 2006a; Côté et al. 2006). Such galaxies typically lack conspicuous central nuclei at their photocenters. The absence of a central excess may suggest that the process of nucleus-building - perhaps through delayed gas inflows and subsequent star formation - has yet to occur in these apparently young galaxies.

Support for programs GO-9401 and GO-10217 was provided through grants from STScI, which is operated by AURA, Inc., under NASA contract NAS5-26555. Additional support for P.C. was provided by NASA LTSA grant NAG5-11714. C.W.C. acknowledges support provided by National Science Council of Taiwan. L.I. acknowledges support from Fondap Center of Astrophysics. M.J.W. acknowledges support through NSF grant AST-0205960. This research has made use of the NASA/IPAC Extragalactic Database (NED) which is operated by the Jet Propulsion Laboratory, California Institute of Technology, under contract with the National Aeronautics and Space Administration. This publication also makes use of data products from the Sloan Digital Sky Survey 
(SDSS). Funding for SDSS and SDSS-II has been provided by the Alfred P. Sloan Foundation, the Participating Institutions, the National Science Foundation, the U.S. Department of Energy, the

National Aeronautics and Space Administration, the Japanese Monbukagakusho, the Max Planck Society, and the Higher Education Funding Council for England.

Facilities: HST(ACS/WFC)

\section{REFERENCES}

Adelman-McCarthy, J., et al. 2007, ApJS, in press, VizieR Online Data Catalog, 2276, 0

Ashman, K.M., Bird, C.M., \& Zepf, S.E. 1994, AJ, 108, 2348

Bahcall, J.N., \& Wolf, R.A. 1976, ApJ, 209, 214

Barnes, J.E., \& Hernquist, L. 1991, ApJ, 370, L65

Bender, R. et al. 1989, A\&A, 217, 35

Binggeli, B., Sandage, A., \& Tammann, G.A. 1985, AJ, 90, 1681

Binggeli, B., \& Jerjen, H. 1998, A\&A, 333, 17

Blanton, M.R., et al. 2003, ApJ, 592, 819

Caon, N., Capaccioli, M. \& D’Onofrio, M. 1993, MNRAS, 265, 1013

Côté, P., et al. 2004, ApJS, 153, 223 (ACSVCS Paper I)

Côté, P., et al. 2006, ApJS, 165, 57 (ACSVCS Paper VIII)

Ebisuzaki, T., Makino, J., \& Okumura, S.K. 1991, Nature, 354, 212

Emsellem, E., et al. 2007, MNRAS, in press, ArXiv Astrophysics e-prints, arXiv:astro-ph/0703531

Faber, S.M., et al. 1997, AJ, 114, 1771

Ferguson, H.C. 1989, AJ, 98, 367

Ferguson, H.C. \& Sandage, A. 1988, AJ, 96, 1520

Ferrarese, L., van den Bosch, F.C., Ford, H.C., Jaffe, W., \& O'Connell, R.W. 1994, AJ, 108, 1598

Ferrarese, L., et al. 2006a, ApJS, 164, 334 (ACSVCS Paper VI)

Ferrarese, L., et al. 2006b, ApJ, 644, L21

Ferrarese, L., et al. 2006c, ArXiv Astrophysics e-prints, arXiv:astro-ph/0612139 
Ford, H.C., et al. 1998, Proc. SPIE, 3356, 234

Fukugita, M., Shimasaku, K., \& Ichikawa, T. 1995, PASP, 107, 945

Graham, A.W. 2004, AJ, 613, L33

Graham, A.W., Erwin, P., Trujillo, I., \& Asensio Ramos, A. 2003, AJ, 125, 2951

Graham, A. W., \& Guzmán, R. 2003, AJ, 125, 2936

Grant, N.I., Kuipers, J.A., \& Phillipps, S. 2005, MNRAS, 363, 1019

Hopkins, P. F., Hernquist, L., Cox, T. J., Robertson, B., \& Krause, E. 2007, ApJ, submitted, ArXiv Astrophysics e-prints, arXiv:astro-ph/0701351

Jordán, A., et al. 2004, ApJS, 154, 509 (ACSVCS Paper II)

Jordán, A., et al. 2007, ApJS, 169, 213 (ACSFCS Paper I)

Kormendy, J. 1985, ApJ, 295, 73

Kormendy, J., \& Djorgovski, S. 1989, ARA\&A, 27, 235

Kormendy, J. 1999, Galaxy Dynamics - A Rutgers Symposium, 182, 124

Lauer, T.R., et al. 1995, AJ, 110, 2622

Lauer, T.R., et al. 2007, ApJ, 664, 226

Li, Y., Haiman, Z., \& Mac Low, M.-M. 2007, ApJ, 663, L61

Loveday, J., Peterson, B.A., Efstathiou, G., \& Maddox, S.J. 1992, ApJ, 390, 338

Marzke, R.O., Huchra, J.P., \& Geller, M.J. 1994, ApJ, 428, 43

McLachlan, G.J., \& Basford, K.E. 1988, Mixture Models: Inference and Application to Clustering (New York: Dekker)

McLaughlin, D.E., King, A.R., \& Nayakshin, S. 2006, ApJ, 650, L37

Mihos, J.C., \& Hernquist, L. 1994, ApJ, 437, L47

Milosavljević, M., \& Merritt, D. 2001, ApJ, 563, 34

Mei, S., et al. 2005, ApJ, 625, 121 (ACSVCS Paper V)

Mei, S., et al. 2007, ApJ, 655, 144 (ACSVCS Paper XIII)

Merritt, D., \& Szell, A. 2006, ApJ, 648, 890 
Nipoti, C., Londrillo, P., \& Ciotti, L. 2006, MNRAS, 370, 681

Ravindranath, S., Ho, L.C., Peng, C.Y., Filippenko, A.V., \& Sargent, W.L.W. 2001, AJ, 122, 653

Rest, A., et al. 2001, AJ, 121, 2431

Rossa, J., et al. 2006, AJ, 132, 1074

Sandage, A., Binggeli, B., \& Tammann, G.A. 1985, AJ, 90, 1759

Sérsic, J.-L. 1968, Atlas de Galaxias Australes (Córdoba: Obs. Astron., Univ. Nac. Córdova)

Schechter, P. 1976, ApJ, 203, 297

Schweizer, F. 1980, ApJ, 237, 303

Stiavelli, M., Miller, B.W., Ferguson, H.C., Mack, J., Whitmore, B.C., \& Lotz, J.M. 2001, AJ, 121, 1385

West, A.A., et al. 2007, AJ, submitted

Verolme, E.K., et al. 2002, MNRAS, 335, 517

Wehner, E.H., \& Harris, W.E. 2006, ApJ, 644, L17

Worthey, G. 2004, AJ, 128, 2826 